\title{
Coupling Chemical Evolution with SPH
}

\author{
Giovanni Carraro \\ Astronomy Department, Padova University, Vicolo Osservatorio 2, \\ I-35122, Padova \\ Laura Portinari \\ Theoretical Astrophysics Center, Juliane Marie Vej 30, DK-2100, \\ Copenhagen
}

\section{Cesario Lia}

SISSA/ISAS, Via Beirut 2, I-34014, Trieste

\begin{abstract}
We present a new statistical algorithm of Chemical Evolution implemented into the Tree-SPH code developed by Lia \& Carraro (2000).
\end{abstract}

In Smoothed Particles Hydrodynamics (SPH) codes with a large number of particles, star formation as well as gas and metal restitution from dying stars can be treated statistically. This approach allows to include detailed chemical evolution and gas re-ejection with minor computational effort. Here we draw the attention on a new statistical algorithm for star formation (SF) and chemical evolution, especially conceived for SPH simulations with large numbers of particles, and for parallel SPH codes described in full detail elsewhere in Lia et al (2001). Basically SF is treated with a probabilistic approach, and when stars are formed they are considered as a single stellar population (SSP) for which gas restitution and metal production for different species ( $\mathrm{He}, \mathrm{Fe}, \mathrm{Ca}, \mathrm{Z}, \mathrm{Mg}, \mathrm{O}, \mathrm{N}, \mathrm{C}, \mathrm{S}$ and total metallicity Z) are computed as a function of the age of the SSP, taking into account the role of single stars of all masses and lifetimes, as well as of Type Ia SN. Our simulations include also Feed-Back from $\mathrm{SNæ}$ of both type Ia and II, and stellar winds. A diffusion mechanism is adopted to spread metals in the interstellar medium (ISM). A novelty of our approach is that also the gas and metals restitution is treated in a probabilistic manner.

We present two astrophysical simulations obtained with this algorithm.

In the first one, we follow the formation of an individual disc-like galaxy, predict the final structure, the metallicity evolution and metallicity gradients. In the left panel of Fig. 1 we present the trend of $[\mathrm{O} / \mathrm{Fe}]$ vs. $[\mathrm{Fe} / \mathrm{H}]$ at the end of the simulation, and compare it with observational data for the solar vicinity.

In the second one we simulate the formation and evolution of a cluster of galaxies, to demonstrate the capabilities of the algorithm in investigating the chemodynamical evolution of the intergalactic medium in a cosmological context. The right panel of Fig. 1 shows the enrichment of the intra-cluster medium in global 

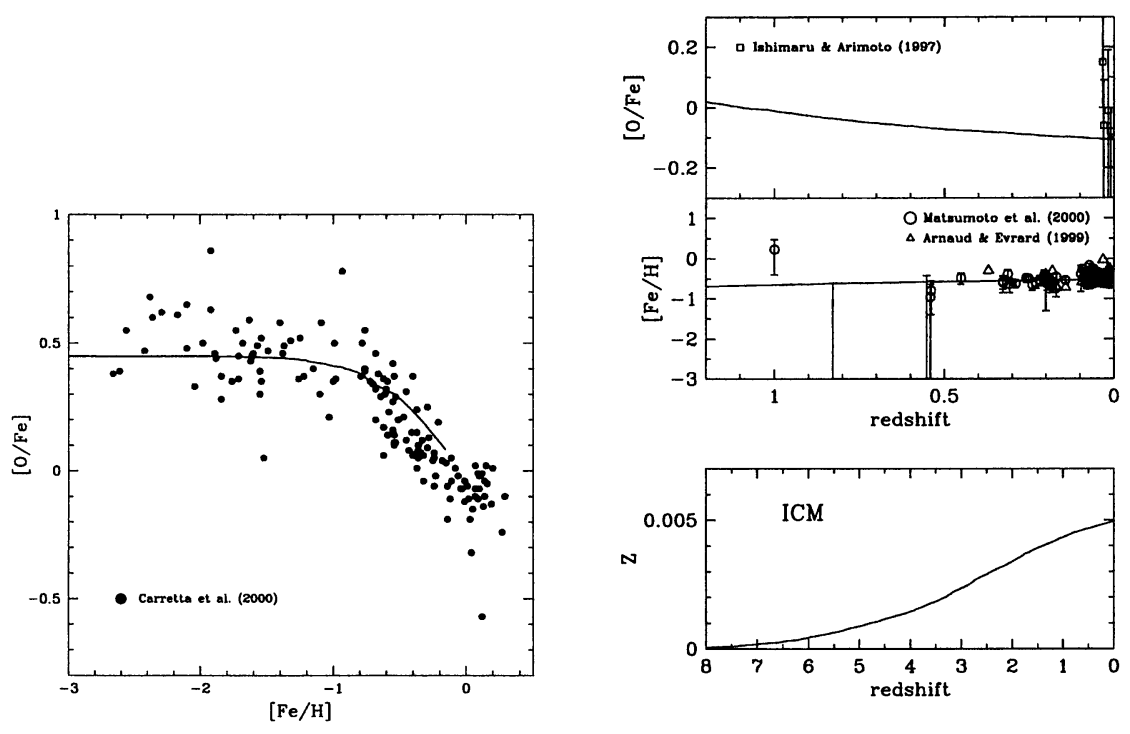

Figure 1. Chemical enrichemnt of a disk galaxy model (left panel) and of the intra-cluster medium (right panle).

metallicity $(\mathrm{Z}),[\mathrm{Fe} / \mathrm{H}]$ and $[\mathrm{O} / \mathrm{Fe}]$. Whenever it is possible, a comparison is made with observational data for cluster of galaxies. As one can note, the agreement is very good.

Our implementation is intended to be easily implemented into any SPH code. Anybody interested is welcome.

Acknowledgments. GC deeply thanks IAU for financial support. The authors acknowledge useful discussions with Jesper Sommer-Larsen and Claudio Dalla Vecchia.

\section{References}

Arnaud M., Evrard A.E., 1999, MNRAS 305, 631

Carretta E., Gratton R.G., Sneden C., 2000, A\&A 356, 238

Ishimaru Y., Arimoto N., 1997, PASJ 49, 1

Kroupa P., 1998, MNRAS 298, 231

Lia C., Carraro G., 2000, MNRAS 314, 145

Lia C., Portinari L., Carraro G., 2001, MNRAS, submitted

Matsumoto H. et al, 2000, PASJ 52, 153 